\title{
Adaptive Laser Beam Shaping with a Linearized Transport-of-Intensity Equation
}

\author{
Leonardo Blanco ${ }^{1}$, Nicolas Védrenne ${ }^{2}$, Vincent Michau ${ }^{2}$, Laurent M. Mugnier ${ }^{2}$, Gilles \\ Chériaux ${ }^{1,3}$ \\ 1: Laboratoire d'Optique Appliquée, ENSTA Paristech, Chemin de la Hunière, 91761 Palaiseau cedex, France \\ 2: Onera, The French Aerospace Lab, 29 Avenue de la Division Leclerc, BP 72, 92322 Châtillon cedex, France \\ 3: Laboratoire pour l'utilisation des lasers intenses, École Polytechnique, 91128 Palaiseau cedex, France \\ leonardo.blanco@ensta-paristech.fr
}

\begin{abstract}
We present a novel method to control the phase and amplitude of a femtosecond laser beam using a linearized version of the transport-of-intensity equation. Simulations show a peak power improvement better than $30 \%$.

(C) 2015 Optical Society of America

OCIS codes: $220.1080,140.3300$
\end{abstract}

\section{Introduction}

In order to maximize the peak power at the focal volume of a high-intensity femtosecond laser beam, phaseconjugation (i.e. correction of the phase aberrations of the beam) with adaptive optics (AO) is not enough. Correction of both the phase and amplitude (field conjugation) [1] is needed. Phase and amplitude control has been a field of research for the last two decades in free-space communications [2] and high-contrast imaging in astronomy [3]. We present a novel method to perform field-conjugation on a femtosecond laser using two deformable mirrors (DM). The method is based on a linearized approach of the transport-of-intensity equation and is performed in a single step, contrary to the iterative Gerchberg-Saxton algorithm and its variations used in most of the litterature.

\section{Amplitude control method}

The problem is to control the amplitude of a laser beam at a plane $\mathrm{P}_{2}$ by controlling the phase of the beam at plane $\mathrm{P}_{1}$, for example with a deformable mirror. To retrieve the phase to be applied at $\mathrm{P}_{1}$, we use the transport-of-intensity equation [4] and we approximate the intensity derivative over $z$ with a finite difference:

$$
\nabla(I \nabla \phi)=-\frac{2 \pi}{\lambda} \frac{\partial}{\partial z} I \approx-\frac{2 \pi}{\lambda} \frac{I_{2}-I_{1}}{\Delta z},
$$

where $\lambda$ is the beam wavelength, $I_{1}$ (respectively $I_{2}$ ) is the beam intensity at plane $\mathrm{P}_{1}$ (respectively $\mathrm{P}_{2}$ ) and $\Delta_{z}$ is the distance between plane $\mathrm{P}_{1}$ and $\mathrm{P}_{2}$. Eq. 1 is linear and can be written in matrix form:

$$
M_{I_{1}}\left(\phi_{0}+\phi_{1}\right)=-\frac{2 \pi}{\lambda} \frac{I_{2}-I_{1}}{\Delta z},
$$

where $\phi_{0}$ is the phase of the beam incident on $\mathrm{DM}_{1}$ and $\phi_{1}$ is the phase applied by $\mathrm{DM}_{1}$.

The solution phase $\hat{\phi}_{1}$ therefore reads:

$$
\hat{\phi}_{1}=-\frac{2 \pi}{\lambda} M_{I_{1}}^{\dagger}\left(I_{2}^{t}-I_{1}\right)-\phi_{0},
$$

where $M_{I_{1}}^{\dagger}$ is the pseudoinverse of matrix $M_{I_{1}}$ and $I_{2}^{t}$ is the desired (target) intensity at plane $\mathrm{P}_{2}$. In order to reduce the number of degrees of freedom of the inverse problem to be solved, the phase $\phi_{1}$ is described as a linear combination of a limited number of phase vectors, e.g. the influence functions of the deformable mirror. $M_{I_{1}}$ can be considered as an interaction matrix. In practice it is computed according to Eq. 2 where $I_{2}$ is registered for each phase vector.

Phase aberrations are corrected with a second deformable mirror at, or conjugated with, plane $\mathrm{P}_{2}$. 


\section{Simulation results}

Simulations were peformed to estimate the performance of the amplitude control method. The initial, uncorrected intensity at plane $P_{1}$ is represented at Fig. 1, left. The target intensity (Fig. 1, middle-left) at plane $\mathrm{P}_{2}$ is constant over the $\mathrm{DM}_{2}$ pupil (to maximize the peak intensity at the focal plane) with a slight apodization so that there is no sharp intensity drop at the pupil edge. Simulation conditions were as follows : $\mathrm{DM}_{1}$ diameter: $5 \mathrm{~cm} ; \lambda=850 \mathrm{~nm}$; propagation distance was set to half the Fresnel distance $F_{d}$ of the DM actuator pitch $a\left(F_{d}=a^{2} / \lambda\right)$ so that the intensity modulation at plane $\mathrm{P}_{2}$ induced by the phase applied at plane $\mathrm{P}_{1}$ is maximum [5]. In our case, this corresponds to a propagation distance of $10 \mathrm{~m}$ for 12 actuators across the DM diameter. The estimated intensity $\hat{I}_{2}$ at plane $\mathrm{P}_{2}$ is obtained by a Fresnel propagation of the estimated field at plane $\mathrm{P}_{1}\left(\hat{\psi}_{1}=A_{1} \exp \left[i\left(\phi_{0}+\hat{\phi}_{1}\right)\right]\right)$.

Fig. 1 shows, on the left, the simulated uncorrected, apodized target and estimated intensities, in the middle, cuts of the uncorrected, target and corrected intensities and, on the right, the estimated solution phase $\hat{\phi}_{1}$ to be applied by $\mathrm{DM}_{1}$. The improvement is clearly visible with the estimated intensity much closer to the target intensity than the uncorrected. The improvement in peak power at the focal plane, assuming a perfectly corrected phase, is $33 \%$.

The estimated phase to be applied by $\mathrm{DM}_{1}$ had a PV amplitude of $2.83 \mu \mathrm{m}$ (optical), well inside the range of available deformable mirrors.

Once the pseudoinverse matrix $M_{I_{1}}^{\dagger}$ is obtained (this can be done offline), the only operation to be performed to compute the phase to apply by $\mathrm{DM}_{1}$ is a matrix product that takes less than 0.02 seconds on a personal computer (128x128 pixel images).
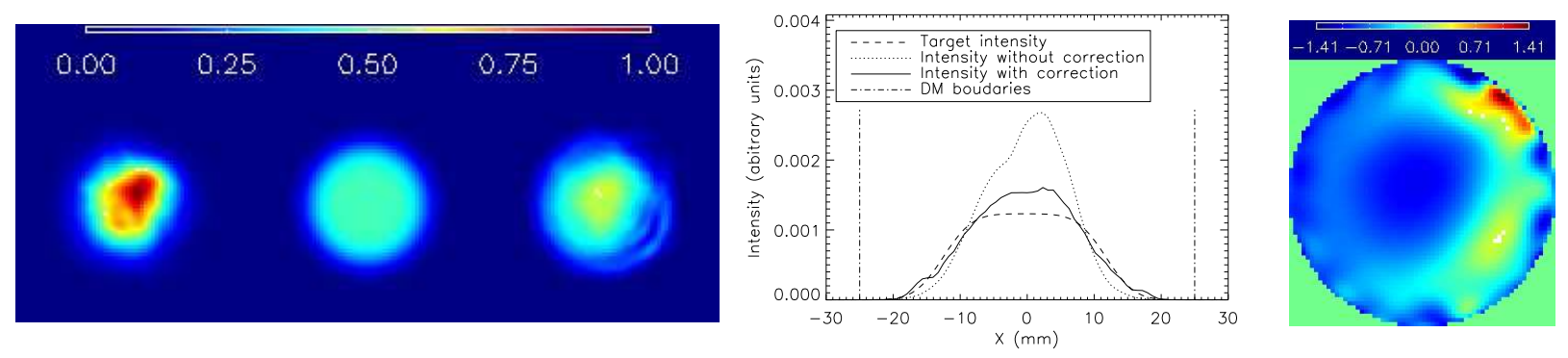

Fig. 1. Left: Uncorrected (at $\left.\mathrm{P}_{1}\right)$, target and corrected (at $\mathrm{P}_{2}$ ) intensities. Middle: cuts of the target (dashed line), uncorrected (dotted line) and corrected (solid line) intensities. Right: estimated phase.

\section{Conclusion}

We have developed a novel method to control the amplitude of a femtosecond laser beam in order to improve the peak power at the focal volume. The method is non-iterative and allows for a quick estimation of the phase to be applied to a deformable mirror to correct for amplitude aberrations in the laser beam. Computer simulations have shown that a better than 30\% increase in peak-power can be expected, which is equivalent to an energy increase of the same amount.

This work is funded by French National Research Agency project ANR-12-ASTR-0008-03.

\section{References}

1. V. P. Lukin F. Y. Kanev. Amplitude phase beam control with the help of a two-mirror adaptive system. Atmospheric and Oceanic Optics, 4:878-881, 1991.

2. Mikhail A. Vorontsov and Valeriy Kolosov. Target-in-the-loop beam control: basic considerations for analysis and wave-front sensing. J. Opt. Soc. Am. A, 22(1):126-141, Jan 2005.

3. F. Malbet, J. W. Yu, and M. Shao. High-Dynamic-Range Imaging Using a Deformable Mirror for Space Coronography. PASP, 107:386, April 1995.

4. Michael Reed Teague. Deterministic phase retrieval: a green's function solution. J. Opt. Soc. Am., 73(11):14341441, Nov 1983.

5. Theodore L. Beach and Richard V. E. Lovelace. Diffraction by a sinusoidal phase screen. Radio Science, 32(3):913-921, 1997. 